\title{
Visceral artery pseudoaneurysm: Is EUS-guided treatment ready for prime time?
}

\section{(1) $\circledast \ominus$}

\author{
Authors \\ Andrea Anderloni ${ }^{1}$, Francesco Auriemma ${ }^{1,2}$, Jason Samarasena ${ }^{3}$
}

Institutions

1 Digestive Endoscopy Unit, Division of Gastroenterology, Humanitas Research Hospital, Milan, Italy

2 Digestive Endoscopy Unit, Humanitas Mater Domini, Castellanza, Italy

3 Division of Gastroenterology and Hepatology, University of California Irvine Medical Center, Orange, California, United States

\author{
Bibliography \\ DOI https://doi.org/10.1055/a-1096-0617 | \\ Endoscopy International Open 2020; 08: E413-E414 \\ (c) Georg Thieme Verlag KG Stuttgart · New York \\ eISSN 2196-9736 \\ Corresponding author \\ Andrea Anderloni, Digestive Endoscopy Unit, Division of \\ Gastroenterology, Humanitas Research Hospital, Milan, \\ Italy 20089 \\ Fax: +02 82247308 \\ andrea.anderloni@humanitas.it
}

Once upon a time...there was a bleeding visceral artery pseudoaneurysm. This extremely rare condition, with an incidence of $0.01 \%$ to $0.2 \%$, usually occurs after vascular injuries or erosions such as in trauma or inflammation [1]. The leading cause is represented by chronic pancreatitis $(C P)$. Sixty-eight percent of visceral artery pseudoaneurysms are secondary to pancreatitis and pseudocyst formation, with up to $17 \%$ of patients with chronic pancreatitis developing pseudoaneurysms. Although prevalence of a pseudoaneurysm in CP is not high, in three of four cases it may bleed, either into the gastrointestinal tract or pancreatic duct, simulating upper gastrointestinal bleeding, or directly into the peritoneal or retroperitoneal cavity, with potentially catastrophic outcomes. Indeed, in the setting of complicated chronic pancreatitis, bleeding has high morbidity and mortality. True visceral aneurysms, in which all three layers are involved, have very low risk of bleeding. False or pseudo-aneurysms arise from a breach of the inner-wall layers, resulting in bulging of the adventitial layer, and carry a very high risk of rupture [2,3].

For both elective and nonelective therapy to date, open and laparoscopic surgical approaches, as well as endovascular interventions have been employed for this condition. In the last 15 years, a few case reports and small case series have appeared in the literature showing the feasibility of endoscopic ultrasound (EUS)-guided obliteration of visceral pseudoaneurysm by means of glue or thrombin injection, alone or combined with coil deployment $[4,5]$.

In this issue of the journal, Maharshi et al. described the technical aspect, outcomes and follow-up of a prospective series of eight patients who underwent EUS-guided thrombin in- jection for management of visceral pseudoaneurysms [6]. Patients enrolled were considered not suitable for an endovascular approach due to technical aspects or economic evaluation. All patients had symptomatic pseudoaneurysms, that is, pain and bleeding. The majority of patients were suffering from complicated CP. Technical success was achieved in all but one case, which required an additional procedure to reach obliteration. No adverse events occurred with a median follow-up of 3 months.

The results demonstrated in this paper are encouraging. Nevertheless, should we add this procedure to the ever-growing list of EUS interventional procedures? Are we really ready for prime time? Despite the lack of specific guidelines, with surgical or endovascular approaches still equally contemplated, according to European Society Gastrointestinal Endoscopy guidelines on endoscopic treatment of chronic pancreatitis (latest update 2018), in case of arterial pseudoaneurysm close to a CP-related pseudocyst, arterial embolization is "strongly" recommended prior to endoscopic drainage, with "low quality of evidence" [7].

Even if not all pseudoaneurysms arise in the setting of chronic pancreatitis, they surely represent the most challenging cases, above all for a surgical approach. Moreover, increasing use of an EUS-guided approach for complications of CP (such as EUS-guided pancreatic fluid collection drainage) especially with introduction of lumen apposing metal stents on the market, has led us to face more situations with potential risk of bleeding from a pseudoaneurysm. EUS-guided obliteration could represent a significant and fashionable alternative to an endovascular route. 
However, there remain many open questions regarding careful evaluation of the pseudoaneurysm's characteristics as it relates to increased embolic risk; use of coils, choice of glue or human thrombin or a combination of more than one. These issues are still not clear in angioembolization practice. Should an EUS-guided approach be the preferred salvage procedure in case of early or delayed rebleeding even if an endovascular route is feasible? Should only false or pseudo-aneurysm be the target or could a true visceral aneurysm be a target, perhaps outside the setting of chronic pancreatitis or another abdominal syndrome? Usually, randomized controlled trials are claimed to resolve open questions in clinical management of controversial entities. In the case of this procedure, there have been too few papers, and too few patients encountered (the current series being the largest, counting eight patients). This warrants at least larger retrospective studies and carefully evaluation before addressing specific and powerful outcomes.

So, ultimately: yes, we may be ready for EUS-guided vascular treatment of visceral artery pseudoaneurysm, but still with a "low quality of evidence."

\section{Competing interests}

The authors declare that they have no conflict of interest.
References

[1] Pulli R, Dorigo W, Troisi N et al. Surgical treatment of visceral artery aneurysms: A 25- year experience. J Vasc Surg 2008; 48: 334-342

[2] Volpi MA, Voliovici E, Pinato F et al. Pseudoaneurysm of the gastroduodenal artery secondary to chronic pancreatitis. Ann Vasc Surg 2010; 24: 1136 e1137-1111

[3] Pitton MB, Dappa E, Jungmann F et al. Visceral artery aneurysms: Incidence, management, and outcome analysis in a tertiary care center over one decade. Eur Radiol 2015; 25: 2004-2014

[4] Roach H, Roberts SA, Salter R et al. Endoscopic ultrasound-guided thrombin injection for the treatment of pancreatic pseudoaneurysm. Endoscopy 2005; 37: 876-878

[5] Rai P, Kc H, Goel A et al. Endoscopic ultrasound-guided coil and glue for treatment of splenic artery pseudo-aneurysm: new kid on the block. Endosc Int Open 2018; 6: E821-E825

[6] Maharshi S, Sharma S, Sharma D et al. Endoscopic ultrasound guided thrombin injection a management approach for visceral artery pseudoaneurysm: case series. Endosc Int Open 2020; 8: E407-E412

[7] Dumonceau JM, Delhaye M, Tringali A et al. Endoscopic treatment of chronic pancreatitis: European Society of Gastrointestinal Endoscopy (ESGE) Guideline - Updated August 2018. Endoscopy 2019; 51: 179193 